\title{
Projections onto convex sets parameter estimation through harmony search and its application for image restoration
}

\author{
Rafael G. Pires ${ }^{1} \cdot$ Danillo R. Pereira $^{1} \cdot$ Luís A. M. Pereira $^{1} \cdot$ Alex F. Mansano $^{1}$ • \\ João P. Papa ${ }^{1}$
}

Published online: 7 July 2015

(C) Springer Science+Business Media Dordrecht 2015

\begin{abstract}
Image restoration is a research field that attempts to recover a blurred and noisy image. Although we have one-step algorithms that are often fast for image restoration, iterative formulations allow a better control of the trade-off between the enhancement of high frequencies (image details) and noise amplification. Projections onto convex sets (POCS) is an iterative-and parametric-based approach that employs a priori knowledge about the blurred image to guide the restoration process, with promising results in different application domains. However, a proper choice of its parameters is a high computational burden task, since they are continuous-valued and there are an infinity of possible values to be checked. In this paper, we propose to optimize POCS parameters by means of harmony search-based techniques, since they provide elegant and simple formulations for optimization problems. The proposed approach has been validated in synthetic and real images, being able to select suitable parameters in a reasonable amount of time.
\end{abstract}

João P. Papa

papa@fc.unesp.br

Rafael G. Pires

rafapires@fc.unesp.br

Danillo R. Pereira

dpereira@ic.unicamp.br

Luís A. M. Pereira

luismartinspr@gmail.com

Alex F. Mansano

afmansano@gmail.com

1 Department of Computing, UNESP - Univ Estadual Paulista, Bauru, Brazil
Keywords Image restoration - Harmony search . Projections onto convex sets

\section{Introduction}

Image processing techniques attempt at enhancing desired properties of an image, with such techniques being widely used for several applications such as preprocessing and postprocessing filters. While geometric techniques aim at correcting distortions and perspective shortcomings, radiometric filters are often used to correct distortions in the image's grey-levels (Gonzalez and Woods 2006). During the acquisition process, the image can get blurred due to the sensor's movement and physical limitations, and also it can become noisy during its transmission. Therefore, the degraded (blurred and noisy) image needs to be processed in order to reduce the noise without loosing the details present in high frequencies. The area of image processing that addresses such issue is called image restoration.

In this context, some well-known image restoration methods, such as the Richardson-Lucy algorithm (1972, 1974) and Wiener filter (1949), for instance, have been widely employed for several applications. Dong et al. (2013) focused on modelling the task of image restoration as a sparse coding problem, and a Markov Random Fieldbased approach was proposed by Chen et al. (2013). Some recent works highlighted image restoration methods based on Total Variation models (Liu et al. 2014; Lv et al. 2013), which exhibit the solution of image denoising and deblurring as minimizers of appropriately chosen functionals.

One of the main problems in one-step image restoration techniques is to restore the image details smoothed by the blurring process with the compromise of keeping the noise in acceptable levels. This fact has motivated the 
development of iterative-based image restoration techniques, in which the trade-off between details and noise may be controlled by means of regularization and projection techniques (Katsaggelos 1991). Since the image restoration problem can be modeled by a linear system, we can employ different approaches to figure out this problem. The Row-Action Projection method (RAP) handles such problem by means of iterative projections onto the hyperplanes defined in the image restoration model (Samaddar and Mammone 1995). This technique belongs to a set of projection-based algorithms commonly addressed by projections onto convex sets (POCS), which use a priori knowledge about the imaging system in order to perform the image restoration task. The key to effectively apply POCS-based algorithms is to define the appropriate sets, and then to compute the projections onto these sets until some criterion be met (Stark et al. 1998).

However, the main limitations of RAP concerns with its relaxation parameter and number of iterations for convergence. The relaxation parameter controls the projections onto the hyperplanes, and high values may turn the restoration process faster, but it is more difficult to handle the artefacts growing process, and low values can lead us to a poorly restored image. The number of iterations strongly affects the restoration process, which means a high value to the relaxation parameter may not require a lot of iterations to restore the image, while low values for that parameter may require more iterations to obtain suitable results. As such, the choice for ideal relaxation and iterations values is extremely dependent on the blur and noise characteristics.

Papa et al. (2010) have modeled the task of finding the relaxation parameter as an optimization problem, in which the fitness function can be some image quality measure. They employed a nature-inspired approach based on Particle Swarm Optimization (PSO) (Kennedy and Eberhart 2001) for such purpose, which has demonstrated a great ability for finding near-optimal relaxation parameters. Later on, Pires et al. (2013) addressed the same problem using the harmony search (HS) technique (Geem 2009), being the results comparable to the ones obtained by means of PSO, but much faster. In this work, we extended both studies with two more contributions: (1) we propose to optimize both the relaxation parameter and number of iterations, since they are strongly dependent on each other, and (2) we evaluated a number of HS-based techniques to find out suitable RAP parameters: harmony search (HS) (Geem 2009), global-best harmony search (Omran and Mahdavi 2008), Improved Harmony Search (Mahdavi et al. 2007), Novel Global Harmony Search (Zou et al. 2010) and Self-adaptive Global best Harmony Search (Pan et al. 2010), as well as we have compared the aforementioned techniques with Fletcher-Reeves Conjugate Method (FR) (Fletcher and Powell 1963; Fletcher 1970), Polak-
Ribiere Conjugate Method (PR) (Polak and Ribiere 1969) and the vanilla Gradient Descent (GD) (Cauchy 1847). Additionally, another contribution of this paper is to employ the Black Holes Algorithm (BHA) (Hatamlou 2013) to optimize HS and its variants in order to avoid an empirical and biased configuration of parameters. However, in order to avoid a possible high computational load concerning this meta-optimization step, we firstly reduced the input image (phantom) by a factor of 4 , and then this new image is degraded with a known blurring and noise models for further restoration using POCS with parameters estimated by the meta-heuristic techniques employed in this work. Soon after, the real degraded image can be restored using the parameter learned with the previous experiment.

The remainder of the paper is organized as follows. Section 2 describes the image restoration problem and the POCS model. Section 3 briefly describes the evolutionary optimization techniques employed in this work. Sections 4 and 5 discuss the methodology and the experimental results, respectively, and Sect. 6 states conclusions and future works.

\section{Image restoration by means of projections onto convex sets}

The image restoration can be understood as a technique used to correct the distortions produced by the imaging systems. The purpose is the restoration of the degraded image using a priori knowledge of the degradation phenomenon. Let $f_{m \times m}$ be an uncorrupted image, $g_{m \times m}$ its degraded version and $n_{m \times m}$ an additive noise. Since the degradation process is linear and space-invariant, it can be described as follows:

$\mathbf{g}=\boldsymbol{H} \mathbf{f}+\mathbf{n}$,

where $\mathbf{g}$, $\mathbf{f}$ and $\mathbf{n}$ stand for $1 \times m^{2}$-dimensional vectors (lexicographical order) representing the images $g, f$ and the additive noise $n$, respectively. In addition, $\boldsymbol{H}_{m^{2} \times m^{2}}$ is the convolutional degradation operator, denoted here as the block-circulant matrix (Katsaggelos 1991), which models the blurring effect. In the absence of noise, Eq. 1 can be written as:

$\mathbf{g}=\boldsymbol{H} \mathbf{f}$,

which can be solved using the well-known Kaczmarz's algorithm (1937) by means of iterative and alternated orthogonal projections onto the hyperplanes defined by the above linear system. Figure 1 illustrates Eq. 2 in order to clarify its content.

Each element of $\mathbf{g}$, say that $g_{p}, p=1,2, \ldots, M^{2}$, is generated as follows: 


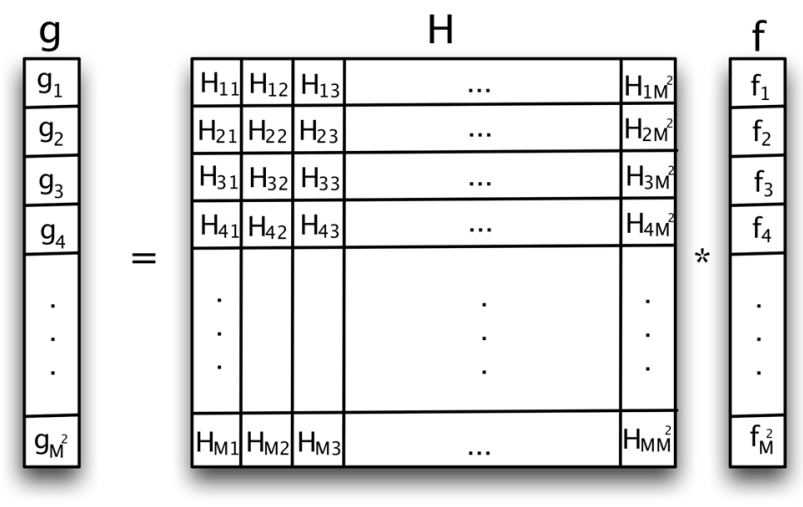

Fig. 1 Image degradation model as a linear and space-invariant system

$g_{p}=\left\langle\mathbf{H}_{p}, \mathbf{f}\right\rangle=\sum_{i=1}^{M^{2}} H_{p i} f_{i}$

Since $\mathbf{g}$ denotes the lexicographical ordering of the degraded image $g$, each element $g_{p}$ stands for a certain pixel in $g$. Therefore, Eq. 3 states that the $p$-th degraded pixel in $\mathbf{g}$ is generated through the inner product between the $p$-th row of $\mathbf{H}$ and the original image in its lexicographical ordering $\mathbf{f}$.

The technique proposed by Kaczmarz to solve Eq. 2 employs the following projecting rule:

$\hat{f}_{p}^{k+1}=\hat{f}_{p}^{k}+\lambda \frac{g_{p}-\left\langle\mathbf{h}_{p}, \hat{f}^{k}\right\rangle}{\left\|\mathbf{h}_{p}\right\|^{2}} \mathbf{h}_{p}$,

where $\lambda$ is the relaxation parameter, which controls the amount of projection, $\mathbf{h}_{p}$ is the $p$-th row of $\mathbf{H}$, i.e., $\mathbf{h}_{p}=\left(H_{p 1}, H_{p 2}, \ldots, H_{p m^{2}}\right)$, and $\hat{f}_{p}^{k}$ stands for the restored image's pixel $p$ at iteration $k, k=1,2, \ldots, K$. Therefore, the idea behind such formulation is to solve the linear system, which means to restore the image. A visualization of the Kaczmarz's algorithm is depicted in Fig. 2, in which we have an example of a linear system with two equations, being the initial guess ("green point") given by $\hat{f}^{0}$ and the final solution ("blue point") represented by $\hat{f}^{K}$. Usually, we employ $\hat{f}^{0}=g$, which means the algorithm is initialized with the degraded image.

The main problem of the Kaczmarz's algorithm regards to the presence of noise, since it has been modeled to solve a linear system like the one described in Eq. 2. However, in practice, we often face the degradation model presented in Eq. 1, which may turn the liner system unstable and without solution, i.e., we have no intersection among the hyperplanes. This fact highlights the importance of choosing proper values for $\lambda$ and the number of iterations $K$, which thus control the trade-off between restoration and noise amplification.

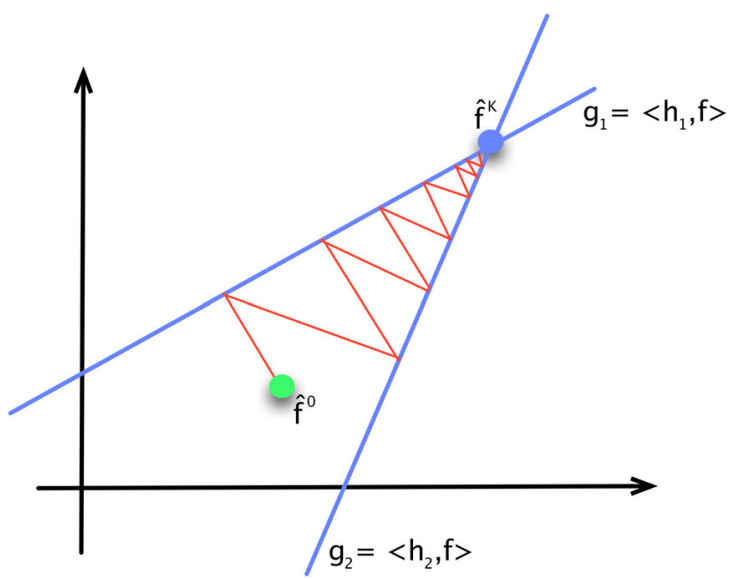

Fig. 2 Graphical representation of the Kaczmarz's algorithm

The Kaczmarz's algorithm is also denoted as the Algebraic Reconstruction Technique (ART) in the field of image reconstruction, or even Row-Action Projection (RAP) in the context of image restoration (Mammone 1992). ${ }^{1}$ The latter one belongs to a class of techniques called projections onto convex sets, which basically aims at projecting the possible solution onto convex sets until their intersection (Stark et al. 1998). In the case of RAP, the convex sets are represented by each hyperplane of the linear system. Another commonly employed convex set is called Limited Amplitude Restriction Set (LA), which basically restricts the pixels' brightness to a range of acceptable values, usually within $[0,255]$. Therefore, the projection onto the set of hyperplanes given by Eq. 4 is usually followed by a projection onto the LA set to correct the grey value of each pixel. This is the procedure adopted in this work.

\section{Meta-heuristic optimization background}

In this section, we briefly review the meta-heuristic optimization techniques employed in this work.

\subsection{Harmony search}

Harmony search (HS) is a meta-heuristic algorithm inspired by the improvisation process of music players. Musicians often improvise the pitches of their instruments searching for a perfect state of harmony (Geem 2009). The main idea is to use the same process adopted by musicians for creating new songs to obtain a near-optimal solution according to some fitness function. Each possible solution

\footnotetext{
${ }^{1}$ Image restoration and reconstruction attempt at the same purpose, they are just used under different names depending on the field of knowledge.
} 
is modeled as a harmony and each musical note corresponds to one decision variable.

The algorithm generates after each iteration a new harmony vector $\mathbf{x}_{\text {new }}=\left(x_{\text {new }}^{1}, x_{\text {new }}^{2}, \ldots, x_{\text {new }}^{N}\right)$ based on memory considerations, pitch adjustments, and randomization (music improvisation), being $N$ the number of decision variables. In regard to the memory consideration step, the idea is to model the process of creating songs, in which the musician can use his/her memories of good musical notes to create a new song. This process is modeled by the Harmony Memory Considering Rate (HMCR) parameter, which is the probability of choosing one value from the historic values stored in the harmony memory, and (1HMCR) is the probability of randomly choosing one feasible value $\theta$ from the range of values $\mathcal{X}_{j}$ of variable $j$, as follows:

$$
x_{\text {new }}^{j}= \begin{cases}x_{A}^{j} & \text { with probability HMCR } \\ \theta \in \mathcal{X}_{j} & \text { with probability (1-HMCR), }\end{cases}
$$

where $A \sim U(1,2, \ldots, P)$, being $P$ the number of harmonies. Further, every component $j$ of the new harmony vector $\mathbf{x}_{n e w}$ is examined to determine whether it should be pitch-adjusted or not, which is controlled by the Pitch Adjusting Rate (PAR) variable, according to Eq. 6:

Pitching adjusting decision for $x_{\text {new }}^{j}$

$$
=\left\{\begin{array}{l}
\text { Yes with probability PAR, } \\
\text { No with probability (1-PAR). }
\end{array}\right.
$$

The pitch adjustment for each instrument is often used to improve solutions and to escape from local optima. This mechanism concerns shifting the neighbouring values of some decision variable in the harmony. As such, if the pitch adjustment decision for the decision variable $x_{\text {new }}^{j}$ is Yes, $x_{\text {new }}^{j}$ is replaced as follows:

$x_{\text {new }}^{j}=x_{\text {new }}^{j} \pm \varphi_{j} \varrho$,

where $\varrho$ is an arbitrary distance bandwidth for the continuous design variable, and $\varphi_{j} \sim U(0,1)$. The next sections describe the variants of harmony search employed in this work.

\subsubsection{Improved harmony search}

The Improved harmony search (IHS) (Mahdavi et al. 2007) differs from traditional HS by updating the PAR and $\varrho$ (bandwidth) values dynamically. The PAR updating formulation at time step $t$ is given by:

$P A R^{t}=P A R_{\min }+\frac{P A R_{\max }-P A R_{\min }}{T} t$,

where $T$ stands for the number of iterations, and $P A R_{\min }$ and $P A R_{\max }$ denote the minimum and maximum number of
PAR values, respectively. In regard to the bandwidth value at time step $t$, it is computed as follows:

$\varrho^{t}=\varrho_{\max } \exp \frac{\ln \left(\varrho_{\min } / \varrho_{\max }\right)}{T} t$,

where $\varrho_{\min }$ and $\varrho_{\max }$ stand for the minimum and maximum values of $\varrho$, respectively.

\subsubsection{Global-best harmony search}

The Global-best Harmony Search (GHS) (Omran and Mahdavi 2008) employs the same modification proposed by IHS with respect to dynamic PAR values. However, it does not employ the concept of bandwidth, being Eq. 7 replaced by:

$x_{\text {new }}^{j}=\hat{x}^{z}$,

where $z \sim U(1,2, \ldots, N)$, and $\hat{\mathbf{x}}$ stands for the best harmony.

\subsubsection{Novel global harmony search}

The Novel Global Harmony Search (NGHS) (Zou et al. 2010) differs from traditional HS in three aspects: (1) the HMCR and PAR parameters are excluded, and a mutation probability $\omega$ is then used; (2) the NGHS always replaces the worst harmony with the new one, and (3) the improvisation footsteps are also modified, as follows:

$R=2 x_{\text {best }}^{j}-x_{\text {worst }}^{j}$,

$x_{\text {new }}^{j}=x_{\text {worst }}^{j}+\mu_{j}\left(R-x_{\text {worst }}^{j}\right)$,

where $\mathbf{x}_{\text {worst }}$ and $\mathbf{x}_{\text {best }}$ stand for the worst and best harmonies, respectively, and $\mu_{j} \sim U(0,1)$. Further, other modification with respect to the mutation probability is performed in the new harmony:

$x_{\text {new }}^{j}= \begin{cases}L^{j}+\varpi_{j}\left(U^{j}-L^{j}\right) & \text { if } \kappa_{j} \leq \omega \\ x_{\text {new }}^{j} & \text { otherwise, }\end{cases}$

where $\kappa_{j}, \varpi_{j} \sim U(0,1)$, and $U^{j}$ and $L^{j}$ stand for the upper and lower bounds of decision variable $j$, respectively

\subsubsection{Self-adaptive global best harmony search}

The SGHS algorithm (Pan et al. 2010) is a modification of the aforementioned GHS, which employs a new improvisation scheme and self-adaptive parameters. First of all, Eq. 10 is rewritten as:

$x_{\text {new }}^{j}=\hat{x}^{j}$,

and Eq. 5 can be replaced by:

$x_{\text {new }}^{j}= \begin{cases}x_{A}^{j} \pm \varphi_{j} \varrho & \text { with probability HMCR } \\ \theta \in \mathcal{X}_{j} & \text { with probability (1-HMCR), }\end{cases}$

in which $A \sim U(1,2, \ldots, P)$. 


\section{Methodology}

In this section we discuss the methodology employed in our work. In short, the POCS-based image restoration algorithm addressed in this paper, works as follows:

1. Set the initial guess as being the degraded image.

2. Project the current guess onto RAP set using Eq. 4.

3. After that, project the image obtained above onto LA set in order to restrict (shrink) the image's pixels within the range $[0,255]$. The obtained image is the current guess.

4. Go to step 2 whether we stopping criterion has not been reached; otherwise finish the algorithm.

The above algorithm gives us a broad idea of the POCS procedure for image restoration: step 2 requires two parameters: the relaxation $\lambda$ value (Eq. 4 ), which controls the amount of projection onto the hyperplanes, ${ }^{2}$ and the number of iterations $K$. In this work we model the task of finding $\lambda$ and $K$ as an optimization problem, since the choice of both parameters is strongly related to the restoration results. Nowadays, it is usual to have images with thousands of hundreds pixels, even in standard cell phones and personal digital cameras, thus being impracticable to perform an exhaustive search for such parameters.

In order to guide the optimization algorithms through the restoration process, Papa et al. (2010) proposed to use some image quality measures as the fitness functions. In the context of image restoration, we can evaluate whether an algorithm achieved suitable results using the Improvement Signal to Noise Ratio (ISNR) (Katsaggelos 1991) or the Universal Image Quality Index (UIQI) (Wang and Bovik 2002), for instance. In this paper, we opted to employ ISNR, since it is a well-accepted quality measure in the image restoration scientific community:

$$
I S N R=10 \log _{10}\left[\frac{(g-f)^{2}}{(\hat{f}-f)^{2}}\right],
$$

in which $\hat{f}$ stands for the restored image. Therefore, the better is the restoration process, the higher is the ISNR. ${ }^{3}$

Let $\Theta=\left(\lambda^{*}, K^{*}\right)$ be the set of parameters that maximized ISNR over the restored image, which has been degraded using some specific degradation process. ${ }^{4}$ Given a real image with similar degradation modeling, we can

\footnotetext{
${ }^{2}$ Under-projections refer to RAP constrained to $\lambda<1$, and overprojections refer to RAP constrained to $\lambda>1$.

3 The better is the quality of the restored image $\hat{f}$, we have that $(\hat{f}-f)^{2} \rightarrow 0$. Thus, $\frac{(g-f)^{2}}{(\hat{f}-f)^{2}} \rightarrow \infty$, which means that high ISNR values stand for better restored images.

${ }^{4}$ The original (uncorrupted) image that has been degraded is the socalled "phantom image".
}

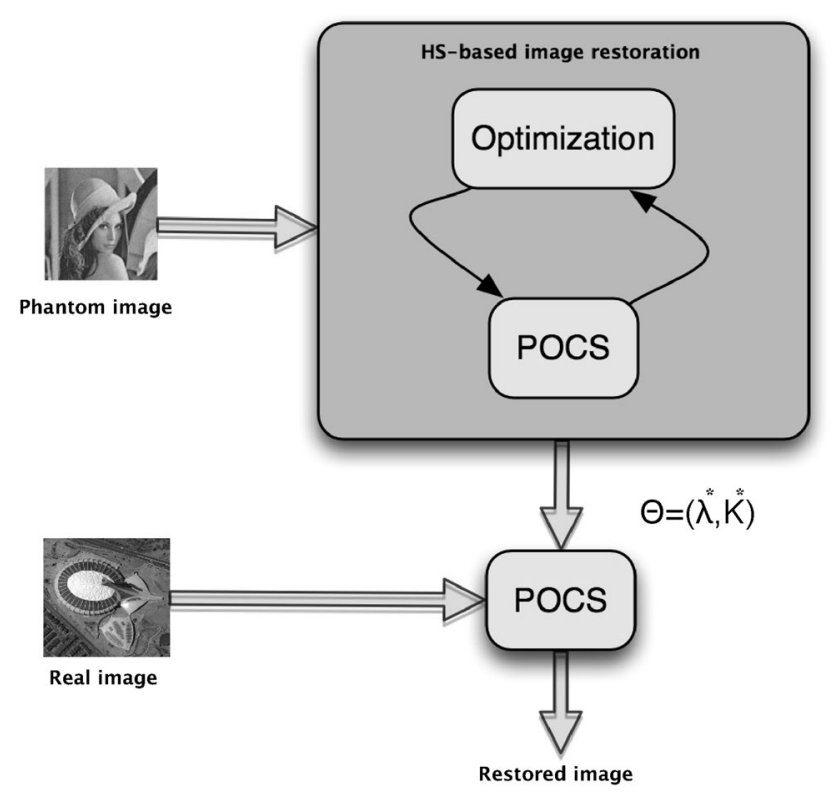

Fig. 3 Proposed pipeline for image restoration

thus employ $\Theta$ to restore such an image. Figure 3 depicts this procedure. Finally, in order to allow a more robust statistical analysis via Wilcoxon signed-rank test (Wilcoxon 1945), we have executed all techniques 10 times, being the initial solutions of GD, FR and PR generated at random. Notice we used the value of 0.05 as the significance level for the statistical test. The statistical analysis considers, for each optimization technique, the ISNR values obtained during the 10 evaluations as the population, being the null hypothesis the one that cogitates that two different optimization techniques achieve the same ISNR value.

In order to avoid possible biases during the parameter setting up, we proposed to meta-optimize HS-based techniques using BHA, which is a parameterless meta-heuristic technique that works similarly to swarm-based techniques, i.e., it updates the whole swarm at all iterations. Therefore, the fitness function used for BHA is the ISNR value obtained through each HS configuration of parameters. ${ }^{5}$ The BHA technique is based on the power of attraction of the black holes, in which each one models a possible solution to the problem. Each black hole has a mass and an amount of charge associated to it, and the gravity force is used for the global search, while the electrical force is used for the local search.

In order to avoid a high computational load during the meta-optimization step, we first reduce the original image by a factor of 4 , for further application of the meta-

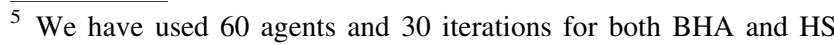
techniques.
} 


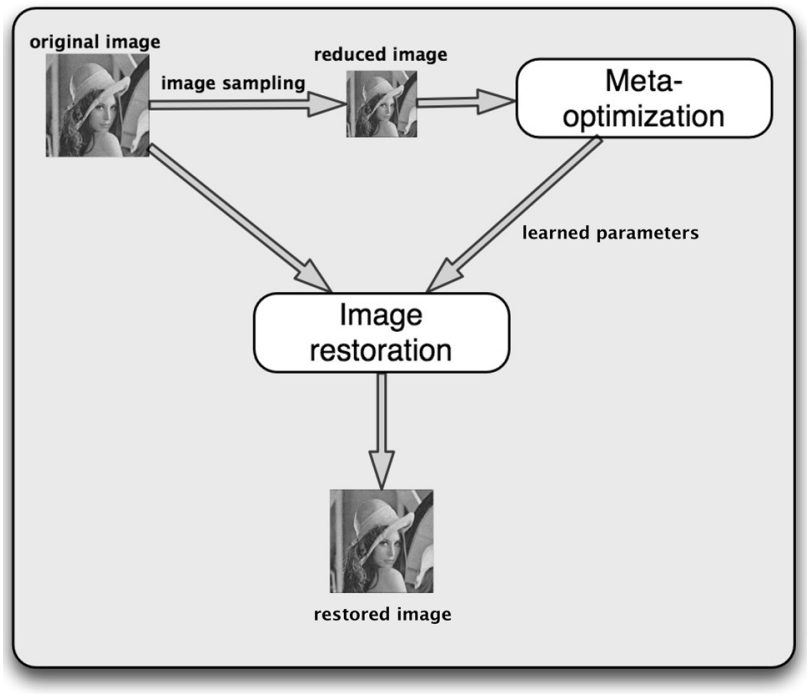

Fig. 4 Proposed pipeline for the meta-optimization step optimization step. Therefore, all fitness evaluations are performed over the reduced image, and the learned set of parameters are then used to restore the original image. This procedure is depicted in Fig. 4.

\section{Experimental results}

In this section we present the experiments conducted to show the effectiveness and efficiency of the meta-heuristic techniques for choosing proper $\lambda$ and $K$ values. The experiments were carried out using the well-known Lena (Fig. 5a) and one remote sensing image (Fig. 6a) in two different situations: the original images have been degraded with a Gaussian blur-GB (Figs. 5b, 6b), as well as with Gaussian blur and additive Gaussian noise- $\mathrm{GB}+\mathrm{N}$ (Figs. 5c, 6c). In regard to the Gaussian blur, we have employed a $3 \times 3$ kernel filter with $\sigma=1.8$. In case of
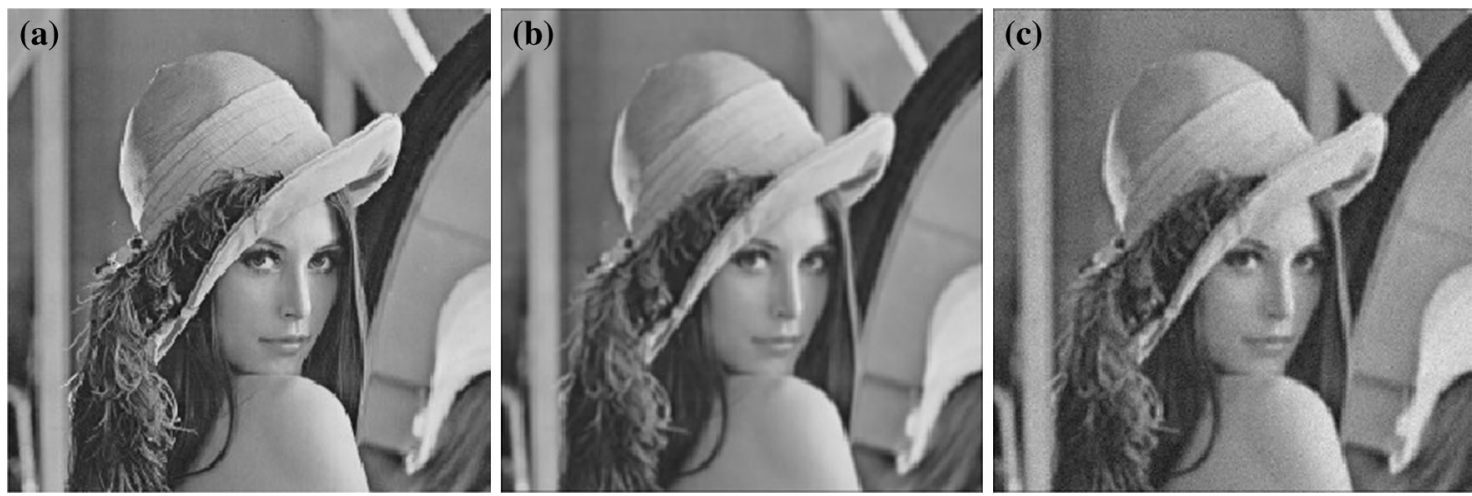

Fig. 5 Lena image with $256 \times 256$ and 8 bits/pixel: a original, b Gaussian blur and c Gaussian blur with additive Gaussian noise
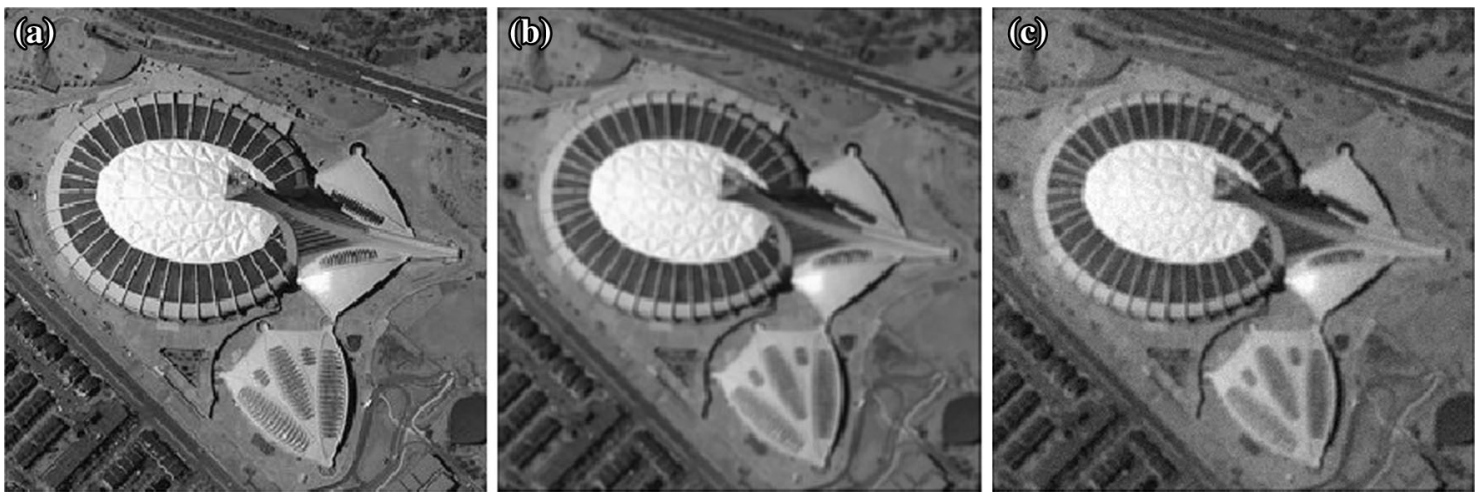

Fig. 6 Remote sensing image 2 with $256 \times 256$ and 8 bits/pixel: a original, b Gaussian blur and c Gaussian blur with additive Gaussian noise 
Table 1 Parameters used for each optimization approach: the number of agents for all techniques was set to 60 , being the number of iterations 30

\begin{tabular}{ll}
\hline Technique & Parameters \\
\hline HS & HMCR $=0.9$, PAR $=0.3$, BW $=0.01$ \\
GHS & HMCR $=0.9$, PARmin $=0.01$, PARmax $=0.99$ \\
IHS & HMCR $=0.9$, PARmin $=0.01$, PARmax $=0.99$ \\
& BWmin $=0.0001$, BWmax $=0.2$ \\
NGHS & $p_{m}=0.033$ \\
SGHS & HMCRm $=0.98$, PARm $=0.9$, BWmin $=0.0005$ \\
& BWmax $=0.4, \mathrm{LP}=2$ \\
\hline
\end{tabular}

Although we have the same variables for different approaches, we decided to keep their original formulation

additive noise, we used with a Gaussian distribution with zero mean and $\sigma=0.0005$.

For each image, we conducted two rounds of experiments: (1) in the first one, a near-exhaustive grid-search has been executed in order to find the optimal values of $\lambda \in$ $[0,2]$ and $K \in[1,20]$ that lead to the best ISNR values; (2) further, the effectiveness of the meta-heuristic techniques in finding suitable $\lambda$ and $K$ values is assessed, since we are interested to know how close the results are to the ones obtained by the near-exhaustive (baseline) search. ${ }^{6}$ Table 1 presents the parameters used for the meta-heuristic techniques, which have been empirically chosen. Notice these parameters are the same for all images.

\subsection{Lena experiments}

This first round of experiments aims at executing the POCS algorithm described in Sect. 4 with $\lambda$ and $K$ obtained by means of a grid-search. Figure 7 displays the ISNR surface obtained by different configurations of $(\lambda, K)$ concerning corrupted Lena Images (Fig. 5b, c). A slower restoration process (Fig. 7b) can be observed with respect to the image corrupted by blurring and noise, since original RAP formulation does not consider the noise effect (Eq. 2). The reader can observe the surface on Fig. $7 \mathrm{~b}$ has a larger area composed by low ISNR values, which are represented by "cold" colors, such as "purple" and "pink".

Further, we employed HS, IHS, GHS, NGHS, SGHS, GD, FR and PR to find out near-optimal values for $\lambda$ and

\footnotetext{
$\overline{6}$ The search range for $\lambda$ was executed within $[0,2]$ with steps of 0.05 , and the search range of $K$ was executed within the range [1, 20] with steps of 1 . This means we have 800 evaluations of the fitness function considering the near-exhaustive search. Although one could meta-optimize the step of grid-search, we do not recommend that, since it might be too costly.
}

$K$. Table 2 presents the quantitative results with respect to ISNR obtained over reduced Lena image, being the best results according to Wilcoxon signed-rank test in bold. We can observe HS-based techniques and PR obtained the best results considering the image with Gaussian blur only (GB), and IHS outperformed all techniques considering the presence of both blurring and noise $(\mathrm{GB}+\mathrm{N})($ Table 3$)$.

In regard to the computational load, Table 4 displays the average number of evaluations to the fitness function (image restoration algorithm) for each approach considered in this work. Since the best results over original Lena image are obtained by means of NGHS and HS, respectively, we may conclude the best trade-off between efficiency and effectiveness is devoted to such techniques. Although we have a slight difference between NGHS and GD in terms of ISNR considering GB experiment, for instance, such difference may rely on dozens of pixels, which may be located at high-frequency regions, thus being of great importance for the perception of detail in images.

Additionally, Table 5 displays the mean computational load for each technique (we are not considering the metaoptimization step for HS, NGHS, SGHS, GHS and IHS). In regard to FR, GD and PR techniques, we also consider a near-exhaustive search to find out the best set of their parameters, i.e., step size $\left(\left[1,10^{-1}, 10^{-2}, \ldots, 10^{-10}\right]\right)$ and the number of iterations $([100, \ldots, 10,000])$. The best value found for the step size was $10^{-4}$, and all techniques converged before 10,000 iterations.

Another interesting point to be considered is related to the meta-optimization step performed by BHA. Since we have 60 agents and 30 iterations for HS-based techniques, they need only $60+30=90$ evaluations of the fitness function. However, as we are using 60 agents for BHA and 30 iterations, it means we need $60 \times 30 \times 90=16,200$ evaluations of the fitness function, which might be timeconsuming (Fig. 8).

\subsection{Remote sensing image experiments}

In this section, we present the experimental results concerning the remote sensing image (Fig. 6). As aforementioned, the main idea is to use the same set of parameters learned over the Lena image to restore this new image, since they are degraded with the same blur and noise models. Table 6 displays the ISNR values for each technique. Considering the blur model only, we can observe the grid-search has obtained the best value, followed by IHS. In regard to both blur and noise degradation, we can observe SGHS as the best technique. Additionally, if we consider this latter model, i.e., blur and noise, HS-based techniques have been consistently better than classical 

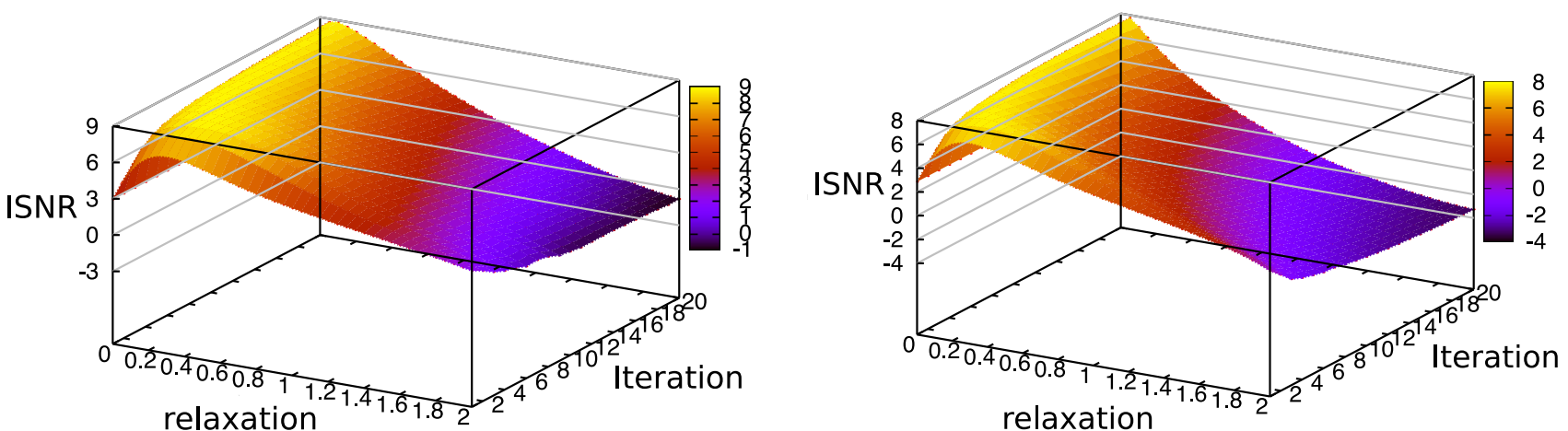

Fig. 7 Grid-search landscape results considering reduced Lena image corrupted by a Gaussian blur (Fig. 5b) and b Gaussian blur and additive noise (Fig. 5c)

Table 2 Restoration results for reduced Lena image considering ISNR for GB and GB+N experiments

\begin{tabular}{lll}
\hline Technique & \multicolumn{2}{l}{ ISNR } \\
\cline { 2 - 3 } & GB & GB $+\mathrm{N}$ \\
\hline Grid-search & $8.9000(0.20)[7]$ & $7.8622(0.25)[3]$ \\
HS & $\mathbf{8 . 9 0 0 3} \pm \mathbf{0 . 0 0 7}(\mathbf{0 . 2 6})[\mathbf{5}]$ & $7.8112 \pm 0.011(0.14)[5]$ \\
NGHS & $\mathbf{8 . 8 9 2 0} \pm \mathbf{0 . 0 2 2}(\mathbf{0 . 2 9})[\mathbf{5}]$ & $7.7785 \pm 0.050(0.15)[6]$ \\
SGHS & $8.8960 \pm 0.014(0.34)[4]$ & $7.8260 \pm 0.022(0.16)[5]$ \\
GHS & $\mathbf{8 . 8 9 7 8} \pm \mathbf{0 . 0 3 9}(\mathbf{0 . 2 8})[\mathbf{5}]$ & $7.8196 \pm 0.028(0.26)[3]$ \\
IHS & $8.8613 \pm 0.028(0.24)[8]$ & $\mathbf{7 . 8 4 4 3}(\mathbf{0 . 1 7})[\mathbf{5}]$ \\
GD & $8.1277 \pm 0.829(0.13)[12]$ & $5.6344 \pm 2.061(0.26)[3]$ \\
FR & $8.8535 \pm 0.058(0.25)[6]$ & $7.4951 \pm 0.653(0.11)[8]$ \\
PR & $\mathbf{8 . 8 7 9 2} \pm \mathbf{0 . 0 3 4}(\mathbf{0 . 2 9})[\mathbf{5}]$ & $7.7522 \pm 0.131(0.25)[8]$ \\
\hline
\end{tabular}

The values in parenthesis stand for the best $\lambda$, and the values in brackets stand for the best $K$. Notice the best value is the one provided by the running that obtained the higher ISNR. The best ISNR values considering the statistical analysis are in bold. The results are displayed in the following format: $x \pm y$, in which $x$ stands for the mean ISNR, and $y$ its standard deviation

Table 3 Restoration results (mean ISNR value) for original Lena image considering ISNR for $\mathrm{GB}$ and $\mathrm{GB}+\mathrm{N}$ experiments

\begin{tabular}{lll}
\hline Technique & \multicolumn{2}{l}{ ISNR } \\
\cline { 2 - 3 } & GB & GB+N \\
\hline Grid-search & 9.5330 & 6.6309 \\
HS & 9.5099 & $\mathbf{6 . 7 6 3 4}$ \\
NGHS & $\mathbf{9 . 5 7 2 5}$ & 6.4514 \\
SGHS & 9.5226 & 6.6101 \\
GHS & 9.4651 & 6.6679 \\
IHS & 9.2633 & 6.5245 \\
GD & 9.5006 & 6.5694 \\
FR & 9.4290 & 6.4946 \\
PR & 9.4228 & 4.4213 \\
\hline The best & ISNR values & are in \\
bold & &
\end{tabular}

optimization techniques. PR, for instance, obtained a very good result over the blurring model only, but a far from suitable result considering blurring and noise. Figure 9 shows the degraded and restored images according to the best techniques in Table 6 .
Table 4 Average number of function calls for each technique

\begin{tabular}{lll}
\hline Technique & \#Calls & \\
\cline { 2 - 3 } & GB & GB+N \\
\hline Grid-search & 800 & 800 \\
HS-based & 90 & 90 \\
GD & 47 & 56 \\
FR & 124.4 & 128 \\
PR & 138.6 & 145.2 \\
BHA & 162,000 & 162,000
\end{tabular}

\section{Conclusions}

Image restoration considers recovering an image degraded by blurring and noise effects. Since it can be modelled as a linear system, several approaches have been proposed to solve the set of equations that leads to suitable restored images. One of such approach is based on projections onto 
Table 5 Mean computational load for learning the POCS parameter

\begin{tabular}{lll}
\hline Technique & \multicolumn{2}{l}{ ISNR } \\
\cline { 2 - 3 } & GB & GB+N \\
\hline Grid-search & $22.48 \mathrm{~h}$ & $22.49 \mathrm{~h}$ \\
HS & $26.83 \mathrm{~h}$ & $24.93 \mathrm{~h}$ \\
NGHS & $17.13 \mathrm{~h}$ & $19.21 \mathrm{~h}$ \\
SGHS & $25.22 \mathrm{~h}$ & $27.38 \mathrm{~h}$ \\
GHS & $28.49 \mathrm{~h}$ & $28.39 \mathrm{~h}$ \\
IHS & $28.42 \mathrm{~h}$ & $29.58 \mathrm{~h}$ \\
FR & $49.1 \mathrm{~min}$ & $59.1 \mathrm{~min}$ \\
GD & $36 \mathrm{~min}$ & $38.5 \mathrm{~min}$ \\
PR & $56 \mathrm{~min}$ & $59.1 \mathrm{~min}$
\end{tabular}
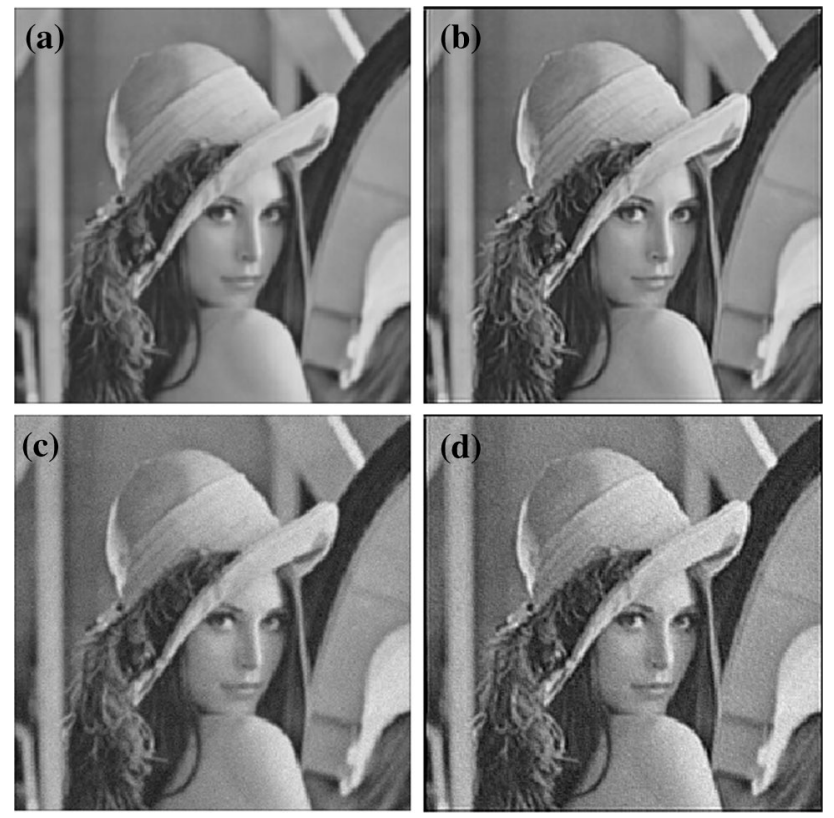

Fig. 8 Degraded Lena images a GB and $\mathbf{c}$ GB $+\mathrm{N}$, and their restored versions using $\mathbf{b}$ NGHS for GB and $\mathbf{d}$ HS for $\mathrm{GB}+\mathrm{N}$

Table 6 Restoration results for Remote Sensing image considering ISNR for GB and GB+N experiments

\begin{tabular}{|c|c|c|}
\hline \multirow[t]{2}{*}{ Technique } & \multicolumn{2}{|l|}{ ISNR } \\
\hline & GB & $\mathrm{GB}+\mathrm{N}$ \\
\hline Grid-search & $8.6314(0.25)[13]$ & $5.3875(0.15)[2]$ \\
\hline HS & $7.9301(0.26)[5]$ & $6.0014(0.14)[5]$ \\
\hline NGHS & $7.7007(0.21)[5]$ & $6.0106(0.15)[6]$ \\
\hline SGHS & $8.0941(0.15)[10]$ & $6.0165(0.16)[5]$ \\
\hline GHS & $8.0000(0.28)[5]$ & $6.0152(0.11)$ [7] \\
\hline IHS & $8.3195(0.24)[8]$ & $6.0127(0.17)[5]$ \\
\hline GD & $8.1383(0.13)[12]$ & $5.9651(0.26)[3]$ \\
\hline FR & $8.1197(0.25)$ [6] & $6.015(0.11)[8]$ \\
\hline PR & $8.0464(0.29)$ [5] & $5.1909(0.25)[8]$ \\
\hline
\end{tabular}

The best ISNR values are in bold
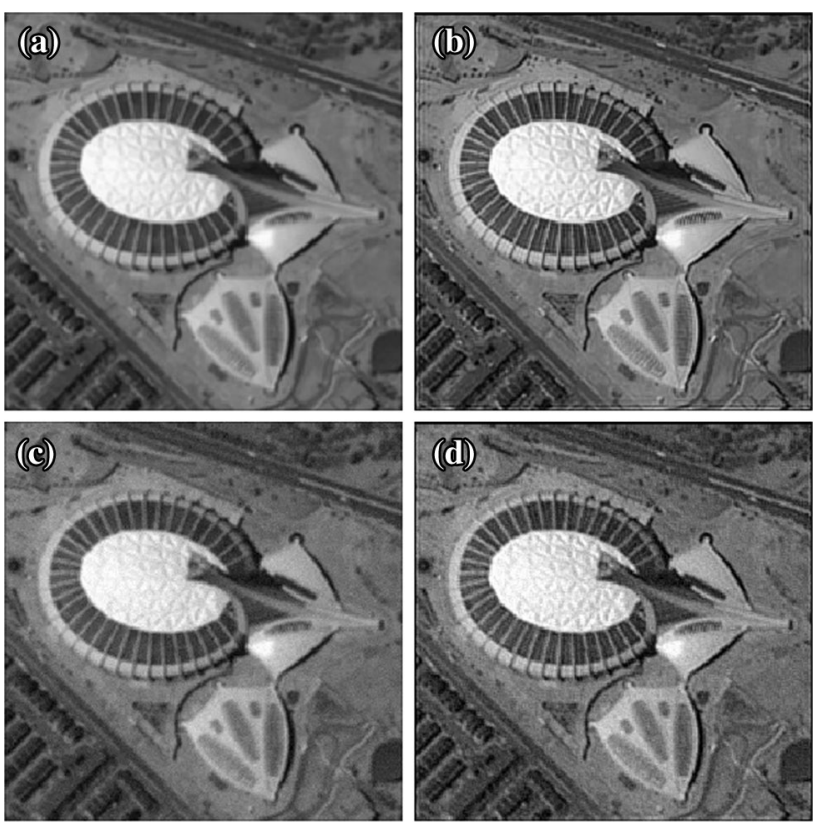

Fig. 9 Degraded remote sensing image a GB and $\mathbf{c} \mathrm{GB}+\mathrm{N}$, and their restored versions using $\mathbf{b}$ grid-search for GB and $\mathbf{d}$ SGHS for $\mathrm{GB}+\mathrm{N}$

convex sets, which employs successive projections in order to iteratively restore the image. The main concern that raises is related to the parameters that control the projection onto the sets: low values may lead us to poorly restored images, and high values can enhance the noise.Therefore, in this paper we propose to find out nearoptimal parameters to guide the projections using several evolutionary optimization techniques. Roughly speaking, the main questions related to this work are oriented to evaluate whether it is reasonable to employ meta-heuristics for optimizing POCS parameters. The answer is positive, but we need to consider the computational load of the meta-optimization process. Our experience shows the meta-optimization procedure conducted over a lower resolution image can suggest parameters to be used in a higher resolution image with similar degradation process.

We conducted two experiments using two images and nine techniques, being a near-exhaustive search (gridsearch), five variants based on the harmony search algorithm, as well as three classical optimization algorithms. In order to fine-tune HS-based approaches, we proposed a meta-optimization schema based on the Black Holes Algorithm, which is a simple and parameterless formulation.

The experiments have shown some important aspects related to the proposed approach: (1) it is possible to learn restoration parameters from one image and then to apply them to restore another image with similar statistics, blur and noise models; (2) HS-based algorithms can obtain similar or even better results than classical optimization algorithms and the grid-search with fewer evaluations of 
the fitness function (in most cases); (3) the proposed metaoptimization approach used in this paper might be used carefully, since it can be time-consuming. In regard to future works, we aim at evaluating different versions of the harmony search algorithm to the context of this work.

Acknowledgments The authors would like to thank Capes and FAPESP Grants \#2009/16206-1, \#2011/14094-1, \#2013/20387-7 and \#2014/16250-9, as well as CNPq Grants \#303182/2011-3, \#470571/ 2013-6 and \#306166/2014-3.

\section{References}

Cauchy AL (1847) Méthode générale pour la résolution des systèmes d'équations simultanées. Compte Rendu des S'eances de L'Acad'emie des Sciences XXV S'erie A(25):536-538

Chen Y, Pock T, Ranftl R, Bischof H (2013) Revisiting loss-specific training of filter-based mrfs for image restoration. In: Weickert $\mathrm{J}$, Hein M, Schiele B (eds) Pattern recognition, lecture notes in computer science, vol. 8142, pp. 271-281. Springer, Berlin. doi:10.1007/978-3-642-40602-7_30

Dong W, Zhang L, Shi G, Li X (2013) Nonlocally centralized sparse representation for image restoration. IEEE Trans Image Process 22(4): $1620-1630$

Fletcher R (1970) A new approach to variable metric algorithms. Comput J 13(3):317-322

Fletcher R, Powell MJD (1963) A rapidly convergent descent method for minimization. Comput J 6(2):163-168

Geem ZW (2009) Music-inspired harmony search algorithm: theory and applications, 1st edn. Springer Publishing Company, Incorporated

Gonzalez R, Woods R (2006) Digital image processing, 3rd edn. Prentice-Hall Inc, Upper Saddle River

Hatamlou A (2013) Black hole: a new heuristic optimization approach for data clustering. Inf Sci 222:175-184

Kaczmarz S (1937) Angenäherte auflösung von systemen linearer gleichungen. Bulletin International de l'Académie Polonaise des Sciences et des Lettres. Classe des Sciences Mathmatiques et Naturelles. Série A, Sciences Mathématiques 35:355-357

Katsaggelos A (1991) Digital image restoration. Springer, New York

Kennedy J, Eberhart R (2001) Swarm intelligence. M. Kaufman, Los Altos

Liu H, Xiong R, Ma S, Fan X, Gao W (2014) Non-local extension of total variation regularization for image restoration. In: IEEE international symposium on circuits and systems, pp 1102-1105
Lucy LB (1974) An iterative technique for the rectification of observed distributions. Astron J 79(6):745-754

Lv XG, Song YZ, Wang SX, Le J (2013) Image restoration with a high-order total variation minimization method. Appl Math Model 37(16-17):8210-8224

Mahdavi M, Fesanghary M, Damangir E (2007) An improved harmony search algorithm for solving optimization problems. Appl Math Comput 188(2):1567-1579

Mammone R (1992) Computation methods of signal recovery and recognition. Wiley, London

Omran MG, Mahdavi M (2008) Global-best harmony search. Appl Math Comput 198(2):643-656

Pan QK, Suganthan P, Tasgetiren MF, Liang J (2010) A self-adaptive global best harmony search algorithm for continuous optimization problems. Appl Math Comput 216(3):830-848

Papa JP, Fonseca LMG, de Carvalho LAS (2010) Projections onto convex sets through particle swarm optimization and its application for remote sensing image restoration. Pattern Recognit Lett 31(13):1876-1886

Pires RG, Pereira LAM, Mansano AF, Papa JP (2013) A hybrid image restoration algorithm based on projections onto convex sets and harmony search. In: IEEE international symposium on circuits and systems, pp 2824-2827

Polak E, Ribiere G (1969) Note sur la convergence de methode de directions conjuguees. ESAIM Math Model Numer Anal 3(R1):35-43

Richardson WH (1972) Bayesian-based iterative method of image restoration. J Opt Soc Am 62(1):55-59

Samaddar S, Mammone R (1995) Image restoration using a row action projection method with adaptative smoothing. Opt Eng 34(4):1147

Stark H, Yang Y, Yang Y (1998) Vector space projections: a numerical approach to signal and image processing, neural nets, and optics. Wiley, London

Wang Z, Bovik A (2002) A universal image quality index. IEEE Signal Process Lett 9(3):81-84

Wiener N (1949) Extrapolation, interpolation, and smoothing of stationary time series. Wiley, London

Wilcoxon F (1945) Individual comparisons by ranking methods. Biom Bull 1(6):80-83

Zou D, Gao L, Wu J, Li S (2010) Novel global harmony search algorithm for unconstrained problems. Neurocomputing 73(1618):3308-3318 